TECHNICAL TRANSACTIONS 6/2019

CIVIL ENGINEERING

DOI: $10.4467 / 2353737$ XCT.19.065.10618

SUBMISSION OF THE FINAL VERSION: $21 / 05 / 2019$

\author{
Olga Dąbrowska (iD) orcid.org/0000-0003-2594-5599 \\ olga.dabrowska@pk.edu.pl \\ Cracow University of Technology, Institute of Structural Mechanics \\ Henryk Ciurej (D) orcid.org/0000-0003-4017-6185 \\ hciurej@agh.edu.pl \\ AGH University of Science and Technology, Faculty of Mining and Geoengineering
}

\title{
SENSITIVITY ANALYSIS OF THE DYNAMIC RESPONSE OF A FRAME. \\ PART I: DiRECT DIFFERENTIATION METHOD
}

ANALIZA WRAŻLIWOŚCI ODPOWIEDZI DYNAMICZNEJ RAMY.
CZĘŚĆ I: METODA BEZPOŚREDNIA ANALIZY WRAŻLIWOŚCI

\begin{abstract}
This paper presents a sensitivity analysis related to the solution of a stationary, linear system of second order equations of motion obtained by the Finite Element Method. The Direct Differentiation Method was presented in this paper. The essence of this method is the explicit differentiation of the system of equations with respect to parameters. As a result, derivatives of vectors and matrices are obtained. The necessary material derivatives of vectors and matrices associated with the simplest 3D beam element are presented. Sensitivity analysis consists in searching for changes in physical quantities in relation to selected parameters. Ultimately, the sensitivity analysis comes down to calculating derivatives of specific functions with respect to parameters. Real and continuous design variables are considered.
\end{abstract}

Keywords: sensitivity analysis, direct differentiation method, explicit differentiation

\section{Streszczenie}

Analiza wrażliwości polega na poszukiwaniu zmian wielkości fizycznych względem wybranych parametrów. Sprowadza się ona do obliczania pochodnych określonych funkcji. W pracy przedstawiono analizę wrażliwości rozwiązania stacjonarnego, liniowego układu równań ruchu drugiego rzędu otrzymanego metodą elementów skończonych. Przedstawiono metodę bezpośrednią analizy wrażliwości. Polega ona na bezpośrednim zróżniczkowaniu równań względem parametrów. W rezultacie uzyskano pochodne wektorów i macierzy. Przedstawiono niezbędne pochodne materialne wektorów i macierzy związanych z najprostszym elementem belki 3D. Rozpatrywano rzeczywiste i ciągle zmienne projektowe.

Słowa kluczowe: analiza wrażliwości, bezpośrednia analiza wrażliwości, pochodne równania ruchu 


\section{Introduction}

Sensitivity analysis consists in searching for changes in physical quantities in relation to changes in selected parameters, which are called design variables, decision variables or free variables. Ultimately, the sensitivity analysis comes down to calculating derivatives of specific functions with respect to parameters [10]. These parameters can determine the configuration of the structure (i.e. its geometry), properties of materials, dimensions or characteristics of cross-sections, etc. A wide discussion about various types of parameters is contained in [10, $16,19]$. If the parameters depicts the geometry of the structure, then the sensitivity analysis is related to the determination of material derivatives [13].

In this paper, only real and continuous design variables are analysed. These variables can be arranged in the form of the vector $\mathbf{h}$ :

$$
\mathbf{h}=\left[h_{1}, \ldots, h_{p}, \ldots, h_{N_{p}}\right], \quad h_{p} \in \mathcal{R},
$$

where $N_{p}$ means the number of design variables.

Determination of derivatives of solutions (i.e. sensitivity analysis) is mostly used in the Optimization [16, 15], Identification, in the Structural Health Monitoring [8, 17, 5], Model Updating $[18,12]$. Obtaining derivatives of solutions also provides valuable information at the design stage, indicating which parameter has the largest impact on the determined values $[6,10]$.

The broad literature has been focused on sensitivity analysis (see basic issues eg. $[13,10]$ ). This analysis is commonly implemented in software dedicated to mechanical problems (see eg. $[16,7])$ - unfortunately, it is less frequently applied in Civil Engineering software. The classic approach is dominated by two methods: Direct Differentiation Method (DDM) and Adjoint System Method. Numerous papers have been devoted to both methods. Additionally, the Virtual Distortion Method [11] and Virtual Load Method [2,3] have been developed. All above methods are applied in both linear and nonlinear problems.

This paper presents a sensitivity analysis related to the solution of a stationary, linear system of second order equations of motion obtained by the Finite Element Method (FEM). The DDM will also be presented. The essence of this method is the explicit differentiation of the system of equations with respect to parameters. As a result, derivatives of vectors and matrices are obtained. The necessary material derivatives of vectors and matrices associated with the simplest 3D beam element are presented in this paper.

Presented approach is parallel to $[1,9,6,14]$.

\section{Second order matrix equation of motion}

Movement of the structure with $N$ dynamic degrees of freedom can be written using the $N$ system of ordinary and stationary differential equations: 


$$
\left\{\begin{array}{l}
\mathbf{M}(\mathbf{h}) \ddot{\mathbf{x}}(t, \mathbf{h})+\mathbf{C}(\mathbf{h}) \dot{\mathbf{x}}(t, \mathbf{h})+\mathbf{K}(\mathbf{h}) \mathbf{x}(t, \mathbf{h})=\mathbf{F}(t, \mathbf{h}), \\
\dot{\mathbf{x}}(0, \mathbf{h})=\mathbf{v}_{0}(\mathbf{h}), \quad \mathbf{x}(0, \mathbf{h})=\mathbf{u}_{0}(\mathbf{h})
\end{array}\right.
$$

where matrix $\mathbf{M}, \mathbf{C}$ and $\mathbf{K}$ are symmetric and $\mathbf{K}$ is positive defined; displacement vector $\mathbf{x}$ dependent on time and design parameters. Similarly, the right hand side $\mathbf{F}(t, \mathbf{h})$ describes the excitation of the system. The dot above vector means differentiation over time, i.e.:

$$
\dot{\mathbf{x}}(t, \mathbf{h})=\frac{\partial \mathbf{x}(t, \mathbf{h})}{\partial t} .
$$

In the equation (2) the dependence of the existing matrices on the parameter vector is marked explicitly. In general, the initial conditions may also depend on design parameters. In the analysis of civil structures, it is often assumed that these conditions are zero:

$$
\mathbf{v}_{0}(\mathbf{h})=\mathbf{0}, \mathbf{u}_{0}(\mathbf{h})=\mathbf{0} .
$$

The common used method of obtaining the equation (2) is FEM [4]. The matrices of the system are global matrices expressed in the global coordinate system after the aggregation process. Typically, boundary conditions are also included in the matrices of the (2) system.

One of the method of considering the boundary conditions of the structure and introducing them into the matrix of the system is reorganization of matrices. The key is isolation and ordering those elements of the displacement vector $\mathbf{x}$ of which absolute displacements are known (equal to zero for fixed supports or non-zero for supports receiving settlements, offsets, etc.). The subvector of known generalized displacements is denoted as $\mathbf{x}_{b}$. The remaining components are ordered in $\mathbf{x}_{s}$ subvector, which is associated with unknown displacements of nodes which do not have contact with a boundary. All matrices and vectors are subject to similar division:

$$
\left[\begin{array}{ll}
\mathbf{M}_{s s} & \mathbf{M}_{s b} \\
\mathbf{M}_{b s} & \mathbf{M}_{b b}
\end{array}\right]\left[\begin{array}{c}
\ddot{\mathbf{x}}_{s} \\
\ddot{\mathbf{x}}_{b}
\end{array}\right]+\left[\begin{array}{cc}
\mathbf{C}_{s s} & \mathbf{C}_{s b} \\
\mathbf{C}_{b s} & \mathbf{C}_{b b}
\end{array}\right]\left[\begin{array}{c}
\dot{\mathbf{x}}_{s} \\
\dot{\mathbf{x}}_{b}
\end{array}\right]+\left[\begin{array}{cc}
\mathbf{K}_{s s} & \mathbf{K}_{s b} \\
\mathbf{K}_{b s} & \mathbf{K}_{b b}
\end{array}\right]\left[\begin{array}{c}
\mathbf{x}_{s} \\
\mathbf{x}_{b}
\end{array}\right]=\left[\begin{array}{c}
\mathbf{P}_{s} \\
\mathbf{P}_{b}-\mathbf{R}_{b}
\end{array}\right],
$$

where for all matrices there is $(\bullet)_{b s}=(\bullet)_{s b}^{T}$ (symmetry). Vector $\mathbf{R}_{b}$ consists of reactions i.e. unknown nodal forces associated with the boundary. By expandig the equation (5) we obtained:

$$
\left\{\begin{array}{l}
\mathbf{M}_{s s} \ddot{\mathbf{x}}_{s}+\mathbf{C}_{s s} \dot{\mathbf{x}}_{s}+\mathbf{K}_{s s} \mathbf{x}_{s}=\mathbf{P}_{s}-\mathbf{M}_{s b} \ddot{\mathbf{x}}_{b}-\mathbf{C}_{s b} \dot{\mathbf{x}}_{b}-\mathbf{K}_{s b} \mathbf{x}_{b}, \\
\mathbf{R}_{b}=\mathbf{P}_{b}-\mathbf{M}_{b b} \ddot{\mathbf{x}}_{b}-\mathbf{C}_{b b} \dot{\mathbf{x}}_{b}-\mathbf{K}_{b b} \mathbf{x}_{b}-\mathbf{M}_{b s} \ddot{\mathbf{x}}_{s}-\mathbf{C}_{b s} \dot{\mathbf{x}}_{s}-\mathbf{K}_{b s} \mathbf{x}_{s} .
\end{array}\right.
$$

The first equation in (6) is a state equation - it allows to calculate unknown displacements $\mathbf{x}_{s}$ of non-boundary nodes of a model, with known nodal loads and nodal support displacements. The second equation in (6) allows to calculate the unknown reactions $\mathbf{R}_{b}$ in support nodes with previously determined components of the vector $\mathbf{x}_{s}$. All occurrences of vectors in (6) are dependent on the $\mathbf{h}$ vector, which is not written for the clarity. 
Equations (6) are the general form of discrete motion equations with any kinematic excitations. Thus, one can solve them, for example, the response of a structure subjected to a seismic excitation to any function $\mathbf{x}_{b}=\mathbf{x}_{b}(t)$ (depending only on time) which is theoretically different for each degree of freedom associated with the boundary. The generality of this rule allows to analyse spatially sprawling structures (long bridges, pipelines, dams, geotechnical objects, etc.) subjected to kinematic excitations caused by a moving seismic wave. It is also possible to design buildings subjected to kinematic excitations caused by trains, road traffic (heavy vehicles or trams) or subway etc.

On the other hand the presence of the $\mathbf{P}_{s}$ component allows to enter any other excitations forces (including harmonics) into free nodes of the mechanical system.

If the boundary conditions are unchanged in time (no movement of supports) and equal to zero (no static settlements), i.e. $\mathbf{x}_{b}(t, \mathbf{h}) \equiv \mathbf{0}$, then the equations (6) take the form:

$$
\left\{\begin{array}{l}
\mathbf{M}_{s s} \ddot{\mathbf{x}}_{s}+\mathbf{C}_{s \mathbf{s}} \dot{\mathbf{x}}_{s}+\mathbf{K}_{s s} \mathbf{x}_{s}=\mathbf{P}_{s}, \\
\mathbf{R}_{b}=\mathbf{P}_{b}-\mathbf{M}_{b s} \ddot{\mathbf{x}}_{s}-\mathbf{C}_{b s} \dot{\mathbf{x}}_{s}-\mathbf{K}_{b s} \mathbf{x}_{s} .
\end{array}\right.
$$

However, if there is no force excitation in the system $\mathbf{P}(t, \mathbf{h}) \equiv \mathbf{0}$ and only kinematic inputs are present, then:

$$
\left\{\begin{array}{l}
\mathbf{M}_{s s} \ddot{\mathbf{x}}_{s}+\mathbf{C}_{s s} \dot{\mathbf{x}}_{s}+\mathbf{K}_{s s} \mathbf{x}_{s}=-\mathbf{M}_{s b} \ddot{\mathbf{x}}_{b}-\mathbf{C}_{s b} \dot{\mathbf{x}}_{b}-\mathbf{K}_{s b} \mathbf{x}_{b} \\
\mathbf{R}_{b}=-\mathbf{M}_{b b} \ddot{\mathbf{x}}_{b}-\mathbf{C}_{b b} \dot{\mathbf{x}}_{b}-\mathbf{K}_{b b} \mathbf{x}_{b}-\mathbf{M}_{b s} \ddot{\mathbf{x}}_{s}-\mathbf{C}_{b s} \dot{\mathbf{x}}_{s}-\mathbf{K}_{b s} \mathbf{x}_{s} .
\end{array}\right.
$$

In the analysis of building structures most often a simpler form of kinematic excitations is assumed - this is connected with the assumption that the movement of the base within the foundations can be treated as a movement of a rigid body which usually has three translational degrees of freedom (or six DOFs including rotation of the base). So, it possible to write two dependencies:

$$
\mathbf{x}_{b}(t, \mathbf{h})=\mathbf{B}(\mathbf{h}) \mathbf{u}_{b}(t), \quad \mathbf{x}_{s}(t, \mathbf{h})=\mathbf{A}(\mathbf{h}) \mathbf{u}_{b}(t)+\mathbf{y}_{s}(t, \mathbf{h}),
$$

where: vector $\mathbf{u}_{b}$ describes the absolute, stiff movement of the base, the matrix $\mathbf{B}$ expresses the purely geometric relationship of motion of nodes of the boundary of the structure with ground movement, vector $\mathbf{y}_{s}$ expresses relative displacements of the free nodes of the structure, matrix $\mathbf{A}$ expresses the purely geometric relationship of motion of free nodes of structure which is result of the motion of rigid ground and is equal to:

$$
\mathbf{A}=-\mathbf{K}_{s s}^{-1} \mathbf{K}_{s b} \mathbf{B} .
$$

Assuming that the damping of the structure is defined by the classical proportional relations $\mathbf{C}=\alpha \mathbf{M}+\beta \mathbf{K}$ and it is small (i.e. $0 \leq \beta \ll \alpha \ll 1$ ) and that low frequencies dominate in excitation, the first equation in (8) takes the form: 


$$
\mathbf{M}_{s s} \ddot{\mathbf{y}}_{s}+\mathbf{C}_{s s} \dot{\mathbf{y}}_{s}+\mathbf{K}_{s s} \mathbf{y}_{s}=-\left(\mathbf{M}_{s b}-\mathbf{M}_{s s} \mathbf{K}_{s s}^{-1} \mathbf{K}_{s b}\right) \mathbf{B} \ddot{\mathbf{u}}_{b} .
$$

Further considerations assume zero initial conditions (4) for (2).

\section{Drect differentiation method}

The essence of the DDM is the direct differentiation of the equation (2) with respect to the parameter $h_{p}$. After differentiation and arrangement, we get:

$$
\mathbf{M} \frac{\mathcal{D} \ddot{\mathbf{x}}}{\mathcal{D} h_{p}}+\mathbf{C} \frac{\mathcal{D} \dot{\mathbf{x}}}{\mathcal{D} h_{p}}+\mathbf{K} \frac{\mathcal{D} \mathbf{x}}{\mathcal{D} h_{p}}=\frac{\mathcal{D} \mathbf{F}}{\mathcal{D} h_{p}}-\left[\frac{\mathcal{D} \mathbf{M}}{\mathcal{D} h_{p}} \ddot{\mathbf{x}}+\frac{\mathcal{D} \mathbf{C}}{\mathcal{D} h_{p}} \dot{\mathbf{x}}+\frac{\mathcal{D} \mathbf{K}}{\mathcal{D} h_{p}} \mathbf{x}\right],
$$

where zero initial conditions were assumed and the symbol $\mathcal{D}(\bullet) / \mathcal{D} h_{p}$ denotes a material derivative of a given quantity.

The introducing of the material derivative [13] is important due to the fact that changing some parameters (e.g. element length) may involve changing the configuration and geometry of the structure, which in turn causes that a material point as a result of this change has a different position in space than before. Thus, the computed spatial derivative would not be expressed as a quantity. From a mathematical point of view, the material derivative of $f=f(t, s(\mathbf{h}), \mathbf{h})$ is a derivative of a composition mapping:

$$
\frac{\mathcal{D} f}{\mathcal{D} h_{p}}=\frac{\partial f}{\partial s} \frac{\partial s}{\partial h_{p}}+\frac{\partial f}{\partial h_{p}},
$$

where the first summand of (13) is associated with the change node location (convection) and the second summand expresses the change of function $f$ at the fixed node position. Due to the rules of differentiating vectors and matrices, the formula (13) is transferred to the components of these objects. If the parameter does not specify the geometry of the structure and specify of properties of material or the cross-section, then the material derivative is equivalent to a simple derivative (no dependence of $f$ on the $s$ variable).

Finally, derivatives $\mathcal{D} \mathbf{M} / \mathcal{D} h_{p}, \mathcal{D C} / \mathcal{D} h_{p}, \mathcal{D K} / \mathcal{D} h_{p}$ in (12) means material derivatives of inertia, damping and stiffness matrix; $\mathcal{D} \mathbf{x} / \mathcal{D} h_{p}$ and $\mathscr{D} \mathbf{F} / \mathcal{D} h_{p}$ denote material derivatives of system response and excitation. The $\mathcal{D} \mathbf{x} / \mathcal{D} h_{p}{ }_{p}$ and its derivatives with respect to time are unknowns in the equation (12). Thus, by comparing (2) and (12) we can see that in order to determine the material derivatives of the response from the equation (12) we should resolve (2) earlier by designating the $\mathbf{x}$ vector and its time derivatives.

From a formal point of view, equations (2) and (12) have a similar structure - they differ only in the right hand side. Therefore, known and typical methods of direct integrating second order equations can be used.

The next two sections present vectors and matrices and their derivatives used in (12). 


\section{Vectors and matrices for 3D beam elements}

The FEM belongs to the basic numerical methods of solving physical problems. In this section element matrices and their derivatives in relation to beam elements are shown. Beams describes straight frame bars (without shearing) in 3D space. Explicit forms of the stiffness, inertia and damping matrices were presented, without reference to the shape functions. The following text presents only the dependencies necessary to obtain derivatives.

The FEM introduces two types of orthogonal and right coordinate systems: the element's local system for each element and one common global system - Fig. 1. Local system with versors $\overrightarrow{\mathbf{i}}_{e}(\mathbf{h}), \overrightarrow{\mathbf{j}}_{e}(\mathbf{h}), \overrightarrow{\mathbf{k}}_{e}(\mathbf{h})$ can be generally a system depending on the parameters $\mathbf{h}$.
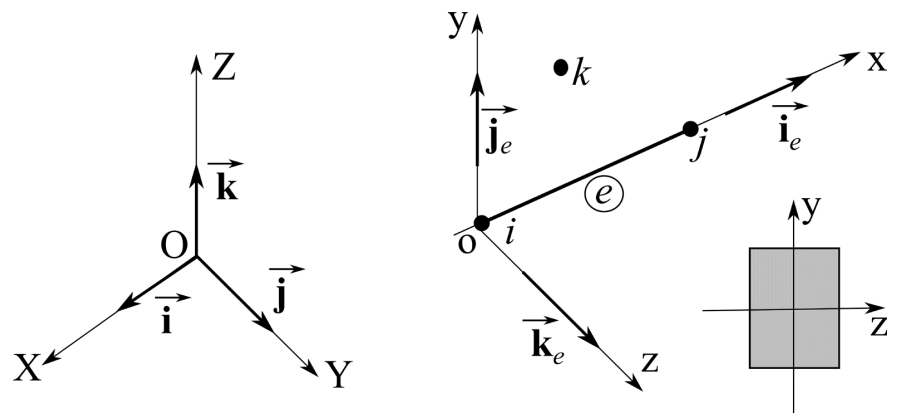

Fig. 1. Coordinate systems: global OXYZ and local 3D beam element oxyz.

Orientation of an exemplary cross-section

A typical beam element $e$ is the line segment between nodes $i, j$. Vectors $\overrightarrow{\mathbf{r}}_{i}(\mathbf{h}), \overrightarrow{\mathbf{r}}_{j}(\mathbf{h})$, specified in the global coordinate system, indicate the location of the nodes. For an unambiguous orientation of the local coordinate system, an additional point $k$ should be introduced with vector $\overrightarrow{\mathbf{r}}_{k}(\mathbf{h})$. Then the versors of the local coordinate system are given the following formulas:

$$
\begin{gathered}
\overrightarrow{\mathbf{i}}_{e}(\mathbf{h})=\frac{\overrightarrow{\mathbf{r}}_{j}(\mathbf{h})-\overrightarrow{\mathbf{r}}_{i}(\mathbf{h})}{L_{e}(\mathbf{h})}, \text { where } L_{e}(\mathbf{h})=\left\|\overrightarrow{\mathbf{r}}_{j}(\mathbf{h})-\overrightarrow{\mathbf{r}}_{i}(\mathbf{h})\right\|, \\
\overrightarrow{\mathbf{k}}_{e}(\mathbf{h})=\frac{\overrightarrow{\mathbf{i}}_{e}(\mathbf{h}) \times\left[\overrightarrow{\mathbf{r}}_{k}(\mathbf{h})-\overrightarrow{\mathbf{r}}_{i}(\mathbf{h})\right]}{\left\|\overrightarrow{\mathbf{i}}_{e}(\mathbf{h}) \times\left[\overrightarrow{\mathbf{r}}_{k}(\mathbf{h})-\overrightarrow{\mathbf{r}}_{i}(\mathbf{h})\right]\right\|}, \\
\overrightarrow{\mathbf{j}}_{e}(\mathbf{h})=\frac{\overrightarrow{\mathbf{k}}_{e}(\mathbf{h}) \times \overrightarrow{\mathbf{i}}_{e}(\mathbf{h})}{\left\|\overrightarrow{\mathbf{k}}_{e}(\mathbf{h}) \times \overrightarrow{\mathbf{i}}_{e}(\mathbf{h})\right\|} .
\end{gathered}
$$

The cross $\times$ means the vector product. As noted above, the vector $\overrightarrow{\mathbf{r}}_{k}(\mathbf{h})$, in general, is dependent on the parameters $\mathbf{h}-$ it means that sensitivity analysis is possible due to the spatial orientation of the element's cross section. This orientation is often associated with parameters defining the geometry of the structure. 
The set of vectors $\overrightarrow{\mathbf{r}}_{l}(\mathbf{h})$, where $l$ iterates through all nodes of the FE model, unambiguously defines the geometry of the structure.

Any vector $\overrightarrow{\mathbf{r}}_{g}$ specified in the global coordinate system OXYZ has coordinates in the local system of the $e$ element defined by vector $\overrightarrow{\mathbf{r}}_{e}$. Both vectors are related by geometric transformation:

$$
\overrightarrow{\mathbf{r}}_{e}(\mathbf{h})=\mathbf{t}_{e}(\mathbf{h}) \overrightarrow{\mathbf{r}}_{g}(\mathbf{h}),
$$

where the transformation matrix $\mathbf{t}_{e}(\mathbf{h})$ is given by:

$$
\mathbf{t}_{e}(\mathbf{h})=\left[\begin{array}{ccc}
\overrightarrow{\mathbf{i}} \circ \overrightarrow{\mathbf{i}}_{e}(\mathbf{h}) & \overrightarrow{\mathbf{j}} \circ \overrightarrow{\mathbf{i}}_{e}(\mathbf{h}) & \overrightarrow{\mathbf{k}} \circ \overrightarrow{\mathbf{i}}_{e}(\mathbf{h}) \\
\overrightarrow{\mathbf{i}} \circ \overrightarrow{\mathbf{j}}_{e}(\mathbf{h}) & \overrightarrow{\mathbf{j}} \circ \overrightarrow{\mathbf{j}}_{e}(\mathbf{h}) & \overrightarrow{\mathbf{k}} \circ \overrightarrow{\mathbf{j}}_{e}(\mathbf{h}) \\
\overrightarrow{\mathbf{i}} \circ \overrightarrow{\mathbf{k}}_{e}(\mathbf{h}) & \overrightarrow{\mathbf{j}} \circ \overrightarrow{\mathbf{k}}_{e}(\mathbf{h}) & \overrightarrow{\mathbf{k}} \circ \overrightarrow{\mathbf{k}}_{e}(\mathbf{h})
\end{array}\right] .
$$

The circle $\circ$ means the dot product. Due to the orthogonality of both coordinate systems, the asymmetric matrix $\mathbf{t}_{e}$ is orthonormal, i.e.:

$$
\mathbf{t}_{e}^{T} \mathbf{t}_{e}=\mathbf{t}_{e}^{-1} \mathbf{t}_{e}=\mathbf{I} .
$$

The nodal forces $\mathbf{f}$ and the moments $\mathbf{m}$ acting in nodes $i, j$ of the beam element $e$ (Fig. 2), can be arranged in the following columns:

$$
\mathbf{f}_{i}^{T}=\left[f_{x i}, f_{y i}, f_{z i}\right], \quad \mathbf{f}_{j}^{T}=\left[f_{x j}, f_{y j}, f_{z j}\right], \quad \mathbf{m}_{i}^{T}=\left[m_{x i}, m_{y i}, m_{z i}\right], \quad \mathbf{m}_{j}^{T}=\left[m_{x j}, m_{y j}, m_{z j}\right] .
$$
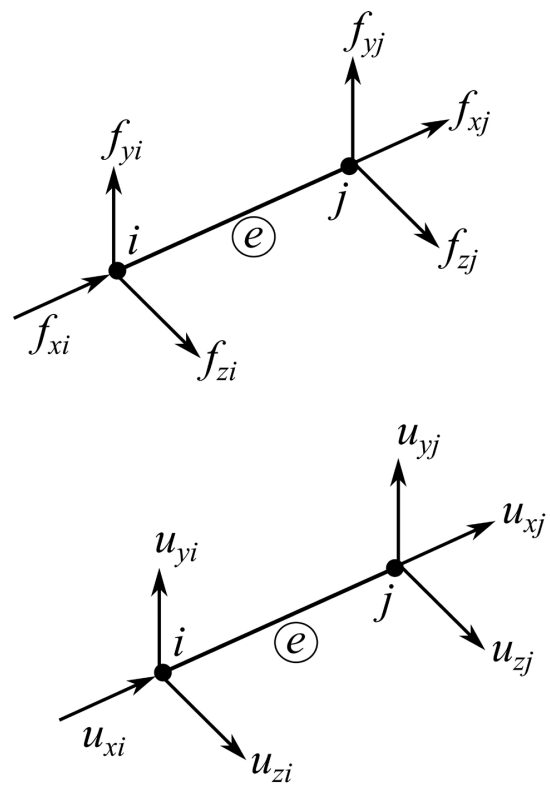
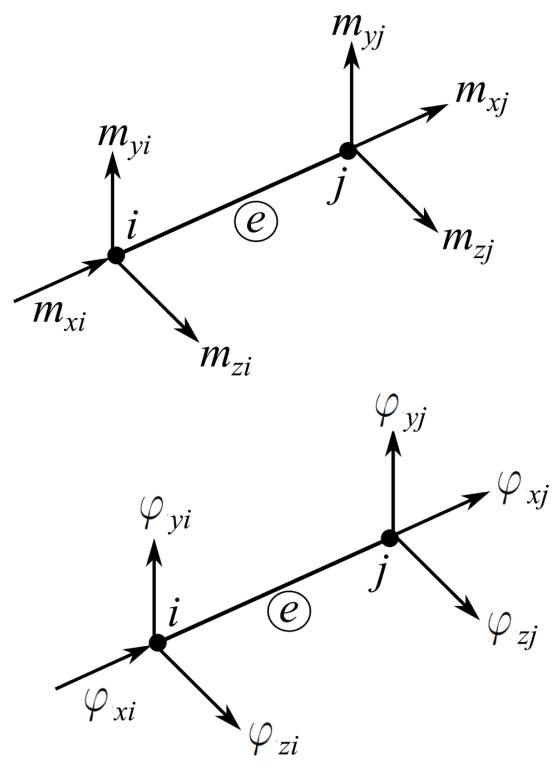

Fig. 2. Presentation of forces $\mathbf{f}$ and moments $\mathbf{m}$ and translation $\mathbf{u}$ and angular $\varphi$ displacements in nodes $i-j$ of finite element $e$ 
Between local and global values based on (17) there is:

$$
\mathbf{p}_{e}(\mathbf{h})=\mathbf{T}_{e}(\mathbf{h}) \mathbf{P}_{e}(\mathbf{h}),
$$

where the dependence of the existing quantities on the $\mathbf{h}$ parameter vector has been marked. Additionally:

$$
\mathbf{p}_{e}^{T}=\left[\mathbf{f}_{i}, \mathbf{m}_{i}, \mathbf{f}_{j}, \mathbf{m}_{j}\right], \quad \mathbf{P}_{e}^{T}=\left[\mathbf{F}_{i}, \mathbf{M}_{i}, \mathbf{F}_{j}, \mathbf{M}_{j}\right], \quad \mathbf{T}_{e}=\operatorname{diag}\left[\mathbf{t}_{e}, \mathbf{t}_{e}, \mathbf{t}_{e}, \mathbf{t}_{e}\right] .
$$

Vectors of forces $\mathbf{F}_{i}, \mathbf{F}_{j}$ and nodal moments $\mathbf{M}_{i}, \mathbf{M}_{j}$ are defined in the global system.

Based on (19), the inverse of the relationship (21) is given by the formula:

$$
\mathbf{P}_{e}(\mathbf{h})=\mathbf{T}_{e}^{-1}(\mathbf{h}) \mathbf{p}_{e}(\mathbf{h})=\mathbf{T}_{e}^{T}(\mathbf{h}) \mathbf{p}_{e}(\mathbf{h}) .
$$

This equation should be used for the aggregation of the node load vector when the $\mathbf{p}_{e}$ vectors are known.

By analogy to (20), the displacements vectors are defined in the $e$ nodes (Fig. 2):

$$
\mathbf{u}_{i}^{T}=\left[u_{x i}, u_{y i}, u_{z i}\right], \quad \mathbf{u}_{j}^{T}=\left[u_{x j}, u_{y j}, u_{z j}\right], \quad \varphi_{i}^{T}=\left[\varphi_{x i}, \varphi_{y i}, \varphi_{z i}\right], \quad \varphi_{j}^{T}=\left[\varphi_{x j}, \varphi_{y j}, \varphi_{z j}\right] .
$$

Similarly to (22), an additional arrangement of displacements is introduced:

$$
\mathbf{u}_{e}^{T}=\left[\mathbf{u}_{i}, \varphi_{i}, \mathbf{u}_{j}, \varphi_{j}\right], \quad \mathbf{x}_{e}^{T}=\left[\mathbf{x}_{i}, \Phi_{i}, \mathbf{x}_{j}, \Phi_{j}\right],
$$

where $\mathbf{u}_{e}$ - are generalized nodal displacements of the element in the local system, $\mathbf{x}_{e}-$ are generalized nodal displacements of the element in the global system. Therefore, for the displacement vectors, the relationship is identical to (21):

$$
\mathbf{u}_{e}(\mathbf{h})=\mathbf{T}_{e}(\mathbf{h}) \mathbf{x}_{e}(\mathbf{h}) .
$$

The physical relation joins the nodal displacement $\mathbf{u}_{e}$ with the nodal forces $\mathbf{p}_{e}$ occurs throughout the element stiffness matrix $\mathbf{k}_{e}$ :

$$
\mathbf{p}_{e}(\mathbf{h})=\mathbf{k}_{e}(\mathbf{h}) \mathbf{u}_{e}(\mathbf{h}),
$$

where matrix $\mathbf{k}_{e}$ has the following structure:

$$
\mathbf{k}_{e}=\frac{E_{e}}{L_{e}^{3}}\left[\begin{array}{cc}
\mathbf{k}_{i i}^{e} & \mathbf{k}_{i j}^{e} \\
\mathbf{k}_{j i}^{e} & \mathbf{k}_{j j}^{e}
\end{array}\right], \quad \mathbf{k}_{i j}^{e}=\left(\mathbf{k}_{j i}^{e}\right)^{T} .
$$

The matrix $\mathbf{k}_{e}$ was defined in the local system. A sequence of formulas for the submatrices appearing in the (28) is presented below:

$$
\mathbf{k}_{i i}^{e}=\left[\begin{array}{ll}
\mathbf{k}_{11}^{e} & \mathbf{k}_{12}^{e} \\
\mathbf{k}_{21}^{e} & \mathbf{k}_{22}^{e}
\end{array}\right], \quad \mathbf{k}_{j j}^{e}=\left[\begin{array}{cc}
\mathbf{k}_{11}^{e} & -\mathbf{k}_{12}^{e} \\
-\mathbf{k}_{21}^{e} & \mathbf{k}_{22}^{e}
\end{array}\right], \quad \mathbf{k}_{j i}^{e}=-\left[\begin{array}{cc}
\mathbf{k}_{11}^{e} & \mathbf{k}_{12}^{e} \\
-\mathbf{k}_{21}^{e} & -\mathbf{k}_{33}^{e}
\end{array}\right], \quad \mathbf{k}_{12}^{e}=\left(\mathbf{k}_{21}^{e}\right)^{T} .
$$


Also:

$$
\begin{gathered}
\mathbf{k}_{11}^{e}=\operatorname{diag}\left[A_{e} L_{e}^{2}, 12 J_{z e}, 12 J_{y e}\right], \quad \mathbf{k}_{22}^{e}=4 L_{e}^{2} \operatorname{diag}\left[\frac{J_{x e}}{8\left(1+v_{e}\right)}, J_{y e}, J_{z e}\right], \\
\mathbf{k}_{21}^{e}=6 L_{e}\left[\begin{array}{ccc}
0 & 0 & 0 \\
0 & 0 & -J_{y e} \\
0 & J_{z e} & 0
\end{array}\right], \quad \mathbf{k}_{33}^{e}=2 L_{e}^{2} \operatorname{diag}\left[\frac{-J_{x e}}{4\left(1+v_{e}\right)}, J_{y e}, J_{z e}\right] .
\end{gathered}
$$

In the entries of the above formulas the dependence on $\mathbf{h}$ was omitted, for the sake of simplicity. An analogous relation to (27) occurs at the element level for objects defined in the global system, i.e.:

$$
\mathbf{K}_{e}(\mathbf{h}) \mathbf{x}_{e}(\mathbf{h})=\mathbf{P}_{e}(\mathbf{h}),
$$

where the stiffness matrix $\mathbf{K}_{e}$ of the element in the global system is related to the matrix $\mathbf{k}_{e}$ by the known transformation

$$
\mathbf{K}_{e}(\mathbf{h})=\mathbf{T}_{e}^{T}(\mathbf{h}) \mathbf{k}_{e}(\mathbf{h}) \mathbf{T}_{e}(\mathbf{h})
$$

The inertia matrix of the element $e$ written in the local system has the following structure:

$$
\mathbf{m}_{e}=\rho_{e}\left[\begin{array}{cc}
\mathbf{m}_{i i}^{e} & \mathbf{m}_{i j}^{e} \\
\mathbf{m}_{j i}^{e} & \mathbf{m}_{j j}^{e}
\end{array}\right], \quad \mathbf{m}_{i j}^{e}=\left(\mathbf{m}_{j i}^{e}\right)^{T} .
$$

The sequence of the formulas containing the submatrices appearing in the (33) is summarized below:

$$
\begin{gathered}
\mathbf{m}_{i i}^{e}=\left[\begin{array}{ll}
\mathbf{m}_{11}^{e} & \mathbf{m}_{12}^{e} \\
\mathbf{m}_{21}^{e} & \mathbf{m}_{22}^{e}
\end{array}\right], \quad \mathbf{m}_{j j}^{e}=\left[\begin{array}{cc}
\mathbf{m}_{11}^{e} & -\mathbf{m}_{12}^{e} \\
-\mathbf{m}_{21}^{e} & \mathbf{m}_{22}^{e}
\end{array}\right], \\
\mathbf{m}_{i j}^{e}=\left[\begin{array}{cc}
\mathbf{m}_{33}^{e} & \mathbf{m}_{34}^{e} \\
-\mathbf{m}_{43}^{e} & -\mathbf{m}_{44}^{e}
\end{array}\right], \quad \mathbf{m}_{12}^{e}=\left(\mathbf{m}_{21}^{e}\right)^{T}, \quad \mathbf{m}_{34}^{e}=\left(\mathbf{m}_{43}^{e}\right)^{T},
\end{gathered}
$$

where:

$$
\begin{gathered}
\mathbf{m}_{11}^{e}=A_{e} L_{e} \operatorname{diag}\left[\frac{1}{3}, \frac{13}{35}, \frac{13}{35}\right]+\frac{6}{5 L_{e}} \operatorname{diag}\left[0, J_{z e}, J_{y e}\right], \\
\mathbf{m}_{22}^{e}=\frac{A_{e} L_{e}^{3}}{105} \operatorname{diag}[0,1,1]+L_{e} \operatorname{diag}\left[\frac{1}{3} J_{x e}, \frac{2}{15} J_{y e}, \frac{2}{15} J_{z e}\right], \\
\mathbf{m}_{12}^{e}=\frac{11 A_{e} L_{e}^{2}}{210}\left[\begin{array}{ccc}
0 & 0 & 0 \\
0 & 0 & 1 \\
0 & -1 & 0
\end{array}\right]+\frac{1}{10}\left[\begin{array}{ccc}
0 & 0 & 0 \\
0 & 0 & J_{z e} \\
0 & -J_{y e} & 0
\end{array}\right],
\end{gathered}
$$




$$
\begin{aligned}
& \mathbf{m}_{33}^{e}=A_{e} L_{e} \operatorname{diag}\left[\frac{1}{6}, \frac{9}{70}, \frac{9}{70}\right]-\frac{6}{5 L_{e}} \operatorname{diag}\left[0, J_{z e}, J_{y e}\right], \\
& \mathbf{m}_{44}^{e}=\frac{A_{e} L_{e}^{3}}{140} \operatorname{diag}[0,1,1]+\frac{L_{e}}{6} \operatorname{diag}\left[-J_{x e}, \frac{1}{5} J_{y e}, \frac{1}{5} J_{z e}\right], \\
& \mathbf{m}_{34}^{e}=\frac{13 A_{e} L_{e}^{2}}{420}\left[\begin{array}{ccc}
0 & 0 & 0 \\
0 & 0 & -1 \\
0 & 1 & 0
\end{array}\right]+\frac{1}{10}\left[\begin{array}{ccc}
0 & 0 & 0 \\
0 & 0 & J_{z e} \\
0 & -J_{y e} & 0
\end{array}\right],
\end{aligned}
$$

The relation of the inertia matrix $\mathbf{M}_{e}$ of the element $e$ written in the global system with the matrix $\mathbf{m}_{e}$ written in the local system of the element is analogous to (32).

The elemental matrices $\mathbf{M}_{e^{\prime}} \mathbf{K}_{e}$ and the vector of nodal loads $\mathbf{P}_{e^{\prime}}$ defined in the global system, constitute the basis for the aggregation of global arrays of the system of equations and global vectors [4]. The general formula of these matrices can be summarized as:

$$
\mathbf{M}=\mathrm{A}_{e} \mathbf{M}_{e}, \quad \mathbf{K}=\mathrm{A}_{e} \mathbf{K}_{e}, \quad \mathbf{P}=\mathrm{A}_{e} \mathbf{P}_{e}
$$

where the $A$ character should be understood in sense of an aggregation operation.

The global damping matrix $\mathbf{C}$ is defined as usual proportional matrix:

$$
\mathbf{C}(\mathbf{h})=\alpha(\mathbf{h}) \mathbf{M}(\mathbf{h})+\beta(\mathbf{h}) \mathbf{K}(\mathbf{h}), \quad 0 \leq \beta \ll \alpha \ll 1 .
$$

\section{Derivatives of vectors and matrices}

Length $L_{e}$ of an element is present in all formulas defining element matrices - hence, it is necessary to derive a derivative of this value with respect to $\mathbf{h}$. The derivative is given by:

$$
\frac{\mathcal{D} L_{e}}{\mathcal{D} h_{p}}=\overrightarrow{\mathbf{i}}_{e} \circ\left(\frac{\mathcal{D} \overrightarrow{\mathbf{r}}_{j e}}{\mathcal{D} h_{p}}-\frac{\mathcal{D} \overrightarrow{\mathbf{r}}_{i e}}{\mathcal{D} h_{p}}\right), \quad h_{p}=1, \ldots, N_{p} .
$$

Derivatives $\mathcal{D} \overrightarrow{\mathbf{r}}_{j e} / \mathcal{D} h_{p}$ and $\mathcal{D} \overrightarrow{\mathbf{r}}_{i e} / \mathcal{D} h_{p}$ of the coordinates of the ends of the bar element must be known and/or calculated before. These derivatives are obtained with known parametrizations of curves or surfaces on which the FE nodes lie.

Derivatives of the versors of the local coordinate system $(14) \div(16)$ are the next functions to be determined:

$$
\begin{gathered}
\frac{\mathcal{D} \overrightarrow{\mathbf{i}}_{e}}{\mathcal{D} h_{p}}=\frac{1}{L_{e}}\left(\frac{\mathcal{D} \overrightarrow{\mathbf{r}}_{j e}}{\mathcal{D} h_{p}}-\frac{\mathcal{D} \overrightarrow{\mathbf{r}}_{i e}}{\mathcal{D} h_{p}}-\overrightarrow{\mathbf{i}_{e}} \frac{\mathcal{D} L_{e}}{\mathcal{D} h_{p}}\right), \\
\overrightarrow{\mathbf{a}}_{e}=\frac{\mathcal{D} \overrightarrow{\mathbf{i}}_{e}}{\mathcal{D} h_{p}} \times\left(\overrightarrow{\mathbf{r}}_{k e}-\overrightarrow{\mathbf{r}}_{i e}\right)+\overrightarrow{\mathbf{i}}_{e} \times\left(\frac{\mathcal{D} \overrightarrow{\mathbf{r}}_{k e}}{\mathcal{D} h_{p}}-\frac{\mathcal{D} \overrightarrow{\mathbf{r}}_{i e}}{\mathcal{D} h_{p}}\right),
\end{gathered}
$$




$$
\begin{gathered}
\frac{\mathcal{D} \overrightarrow{\mathbf{k}}_{e}}{\mathcal{D} h_{p}}=\frac{\overrightarrow{\mathbf{a}}_{e}-\overrightarrow{\mathbf{k}}_{e}\left(\overrightarrow{\mathbf{k}}_{e} \circ \overrightarrow{\mathbf{a}}_{e}\right)}{\left\|\overrightarrow{\mathbf{i}}_{e} \times\left(\overrightarrow{\mathbf{r}}_{k e}-\overrightarrow{\mathbf{r}}_{i e}\right)\right\|}, \\
\overrightarrow{\mathbf{b}}_{e}=\frac{\mathcal{D} \overrightarrow{\mathbf{k}}_{e}}{\mathcal{D} h_{p}} \times \overrightarrow{\mathbf{i}}_{e}+\overrightarrow{\mathbf{k}}_{e} \times \frac{\mathcal{D} \overrightarrow{\mathbf{i}}_{e}}{\mathcal{D} h_{p}}, \\
\frac{\mathcal{D} \overrightarrow{\mathbf{j}}_{e}}{\mathcal{D} h_{p}}=\frac{\overrightarrow{\mathbf{b}}_{e}-\overrightarrow{\mathbf{j}}_{e}\left(\overrightarrow{\mathbf{j}}_{e} \circ \overrightarrow{\mathbf{b}}_{e}\right)}{\left\|\left(\overrightarrow{\mathbf{k}}_{e} \times \overrightarrow{\mathbf{i}}_{e}\right)\right\|} .
\end{gathered}
$$

Now, having derivatives of the versors of the local coordinate system, the derivatives of the transformation matrix $\mathbf{t}_{e}$ and $\mathbf{T}_{e}$ should be calculated. The derivative of the matrix $\mathbf{T}_{e}$ allows to specify the bar stiffness changes in the global coordinate system due to the change of the configuration described by the parameters $h_{p}$. The derivation of $\mathcal{D} \mathbf{T}_{e} / D h_{p}$ reduced to the calculation of $\mathcal{D} \mathbf{t}_{e} / \mathcal{D} h_{p}$ using equations (18) and (22). Thus:

$$
\frac{\mathcal{D} \mathbf{t}_{e}}{\mathcal{D} h_{p}}=\left[\begin{array}{lll}
\overrightarrow{\mathbf{i}} \circ \frac{\mathcal{D} \overrightarrow{\mathbf{i}}_{e}}{\mathcal{D} h_{p}} & \overrightarrow{\mathbf{j}} \circ \frac{\mathcal{D} \overrightarrow{\mathbf{i}}_{e}}{\mathcal{D} h_{p}} & \overrightarrow{\mathbf{k}} \circ \frac{\mathcal{D} \overrightarrow{\mathbf{i}}_{e}}{\mathcal{D} h_{p}} \\
\overrightarrow{\mathbf{i}} \circ \frac{\mathcal{D} \overrightarrow{\mathbf{j}}_{e}}{\mathcal{D} h_{p}} & \overrightarrow{\mathbf{j}} \circ \frac{\mathcal{D} \overrightarrow{\mathbf{j}}_{e}}{\mathcal{D} h_{p}} & \overrightarrow{\mathbf{k}} \circ \frac{\mathcal{D} \overrightarrow{\mathbf{j}}_{e}}{\mathcal{D} h_{p}} \\
\overrightarrow{\mathbf{i}} \circ \frac{\mathcal{D} \overrightarrow{\mathbf{k}}_{e}}{\mathcal{D} h_{p}} & \overrightarrow{\mathbf{j}} \circ \frac{\mathcal{D} \overrightarrow{\mathbf{k}}_{e}}{\mathcal{D} h_{p}} & \overrightarrow{\mathbf{k}} \circ \frac{\mathcal{D} \overrightarrow{\mathbf{k}}_{e}}{\mathcal{D} h_{p}}
\end{array}\right], \frac{\mathcal{D} \mathbf{T}_{e}}{\mathcal{D} h_{p}}=\operatorname{diag}\left[\frac{\mathcal{D} \mathbf{t}_{e}}{\mathcal{D} h_{p}}, \frac{\mathcal{D} \mathbf{t}_{e}}{\mathcal{D} h_{p}}, \frac{\mathcal{D} \mathbf{t}_{e}}{\mathcal{D} h_{p}}, \frac{\mathcal{D} \mathbf{t}_{e}}{\mathcal{D} h_{p}}\right] .
$$

Shown derivatives express that in the most complicated case, when the $h_{p}$ describes the configuration of the structure, non-zero derivatives remain dependencies indirectly or directly on vectors of the ends of the element $e$, i.e. from $\overrightarrow{\mathbf{r}}_{i e}, \overrightarrow{\mathbf{r}}_{j e}$ and $\overrightarrow{\mathbf{r}}_{k e}$. Thus, non-zero derivatives remains of transformation matrix $\mathcal{D} \mathbf{t}_{e} / \mathcal{D} h_{p}, \mathcal{D} \mathbf{T}_{e} / \mathcal{D} h_{p}$ and above all $\mathcal{D} L_{e} / \mathcal{D} h_{p}$. It is also important that the arrays of derivatives in (42) often lose their orthogonality.

The derivation of the stiffness matrix $\mathbf{k}_{e}$ in the local system with respect to $h_{p}$ is defined by the following formulas:

$$
\frac{\mathcal{D} \mathbf{k}_{e}}{\mathcal{D} h_{p}}=\left[\frac{1}{E_{e}} \frac{\partial E_{e}}{\partial h_{p}}-\frac{3}{L_{e}} \frac{\mathcal{D} L_{e}}{\mathcal{D} h_{p}}\right] \mathbf{k}_{e}+\frac{E_{e}}{L_{e}^{3}}\left[\begin{array}{cc}
\frac{\mathcal{D} \mathbf{k}_{i i}^{e}}{\mathcal{D} h_{p}} & \frac{\mathcal{D} \mathbf{k}_{i j}^{e}}{\mathcal{D} h_{p}} \\
\frac{\mathcal{D} \mathbf{k}_{j i}^{e}}{\mathcal{D} h_{p}} & \frac{\mathcal{D} \mathbf{k}_{i j}^{e}}{\mathcal{D} h_{p}}
\end{array}\right], \frac{\mathcal{D} \mathbf{k}_{i j}^{e}}{\mathcal{D} h_{p}}=\left(\frac{\mathcal{D} \mathbf{k}_{j i}^{e}}{\mathcal{D} h_{p}}\right)^{T}, h_{p}=1, \ldots, N_{p}
$$


where:

$$
\begin{gathered}
\frac{\mathcal{D} \mathbf{k}_{i i}^{e}}{\mathcal{D} h_{p}}=\left[\begin{array}{cc}
\frac{\mathcal{D} \mathbf{k}_{11}}{\mathcal{D} h_{p}} & \frac{\mathcal{D} \mathbf{k}_{12}}{\mathcal{D} h_{p}} \\
\frac{\mathcal{D} \mathbf{k}_{21}}{\mathcal{D} h_{p}} & \frac{\mathcal{D} \mathbf{k}_{22}}{\mathcal{D} h_{p}}
\end{array}\right], \frac{\mathcal{D} \mathbf{k}_{j j}^{e}}{\mathcal{D} h_{p}}=\left[\begin{array}{cc}
\frac{\mathcal{D} \mathbf{k}_{11}}{\mathcal{D} h_{p}} & -\frac{\mathcal{D} \mathbf{k}_{12}}{\mathcal{D} h_{p}} \\
-\frac{\mathcal{D} \mathbf{k}_{21}}{\mathcal{D} h_{p}} & \frac{\mathcal{D} \mathbf{k}_{22}}{\mathcal{D} h_{p}}
\end{array}\right], \\
\frac{\mathcal{D} \mathbf{k}_{j i}^{e}}{\mathcal{D} h_{p}}=-\left[\begin{array}{cc}
\frac{\mathcal{D} \mathbf{k}_{11}}{\mathcal{D} h_{p}} & \frac{\mathcal{D} \mathbf{k}_{12}}{\mathcal{D} h_{p}} \\
-\frac{\mathcal{D} \mathbf{k}_{21}}{\mathcal{D} h_{p}} & \frac{\mathcal{D} \mathbf{k}_{33}}{\mathcal{D} h_{p}}
\end{array}\right], \quad \frac{\mathcal{D} \mathbf{k}_{21}}{\mathcal{D} h_{p}}=\left(\frac{\mathcal{D} \mathbf{k}_{12}}{\mathcal{D} h_{p}}\right)^{T} .
\end{gathered}
$$

Additionally:

$$
\begin{gathered}
\frac{\mathcal{D} \mathbf{k}_{11}}{\mathcal{D} h_{p}}=\operatorname{diag}\left[L_{e}\left(\frac{\partial A_{e}}{\partial h_{p}} L_{e}+2 A_{e} \frac{\mathcal{D} L_{e}}{\mathcal{D} h_{p}}\right), 12 \frac{\partial J_{z e}}{\partial h_{p}}, 12 \frac{\partial J_{y e}}{\partial h_{p}}\right], \\
\frac{\mathcal{D} \mathbf{k}_{22}}{\mathcal{D} h_{p}}=\frac{2}{L_{e}} \frac{\mathcal{D} L_{e}}{\mathcal{D} h_{p}} \mathbf{k}_{22}+4 L_{e}^{2} \operatorname{diag}\left[\frac{1}{8\left(1+v_{e}\right)} \frac{\partial J_{x e}}{\partial h_{p}}, \frac{\partial J_{y e}}{\partial h_{p}}, \frac{\partial J_{z e}}{\partial h_{p}}\right], \\
\frac{\mathcal{D} \mathbf{k}_{21}}{\mathcal{D} h_{p}}=\frac{1}{L_{e}} \frac{\mathcal{D} L_{e}}{\mathcal{D} h_{p}} \mathbf{k}_{21}+6 L_{e}\left[\begin{array}{ccc}
0 & 0 & 0 \\
0 & 0 & -\frac{\partial J_{y e}}{\partial h_{p}} \\
0 & \frac{\partial J_{z e}}{\partial h_{p}} & 0
\end{array}\right], \\
\frac{\mathcal{D} \mathbf{k}_{33}}{\mathcal{D} h_{p}}=\frac{2}{L_{e}} \frac{\mathcal{D} L_{e}}{\mathcal{D} h_{p}} \mathbf{k}_{33}+2 L_{e}^{2} \operatorname{diag}\left[\begin{array}{cc}
-\frac{1}{4\left(1+v_{e}\right)} \frac{\partial J_{x e}}{\partial h_{p}}, \frac{\partial J_{y e}}{\partial h_{p}}, \frac{\partial J_{z e}}{\partial h_{p}}
\end{array}\right] .
\end{gathered}
$$

It was assumed that Poisson's ratio $v_{e}$ does not depend on the $h_{p}$ which is justified in practices. Matrix of the derivatives $\mathcal{D} \mathbf{k}_{e} / \mathcal{D} h_{p}$ is symmetrical, but often it is not positively defined (negative values and zeros may happen on the diagonal).

The derivative of the stiffness matrix of an element in the global system is given by the formula:

$$
\frac{\mathcal{D} \mathbf{k}_{e}}{\mathcal{D} h_{p}}=\left(\frac{\mathcal{D} \mathbf{T}_{e}}{\mathcal{D} h_{p}}\right)^{T} \mathbf{k}_{e} \mathbf{T}_{e}+\mathbf{T}_{e}^{T} \frac{\mathcal{D} \mathbf{k}_{e}}{\mathcal{D} h_{p}} \mathbf{T}_{e}+\mathbf{T}_{e}^{T} \mathbf{k}_{e} \frac{\mathcal{D} \mathbf{T}_{e}}{\mathcal{D} h_{p}}, h_{p}=1, \ldots, N_{p} .
$$

where the dependence of (32) and equality 


$$
\left(\frac{\mathcal{D} \mathbf{T}_{e}}{\mathcal{D} h_{p}}\right)^{T}=\frac{\mathcal{D} \mathbf{T}_{e}^{T}}{\mathcal{D} h_{p}}
$$

was utilized.

The derivative of the inertia matrix $\mathbf{m}_{e}$ of the element $e$ in the local coordinate system with respect to $h_{p}$ is expressed by the following formulas:

$$
\frac{\mathcal{D} \mathbf{m}_{e}}{\mathcal{D} h_{p}}=\frac{1}{\rho_{e}} \frac{\partial \rho_{e}}{\partial h_{p}} \mathbf{m}_{e}+\rho_{e}\left[\begin{array}{cc}
\frac{\mathcal{D} \mathbf{m}_{i i}^{e}}{\mathcal{D} h_{p}} & \frac{\mathcal{D} \mathbf{m}_{i j}^{e}}{\mathcal{D} h_{p}} \\
\frac{\mathcal{D} \mathbf{m}_{j i}^{e}}{\mathcal{D} h_{p}} & \frac{\mathcal{D} \mathbf{m}_{j j}^{e}}{\mathcal{D} h_{p}}
\end{array}\right], \frac{\mathcal{D} \mathbf{m}_{i j}^{e}}{\mathcal{D} h_{p}}=\left(\frac{\mathcal{D} \mathbf{m}_{j i}^{e}}{\mathcal{D} h_{p}}\right)^{T}, h_{p}=1, \ldots, N_{p} .
$$

where:

$$
\begin{gathered}
\frac{\mathcal{D} \mathbf{m}_{i i}^{e}}{\mathcal{D} h_{p}}=\left[\begin{array}{cc}
\frac{\mathcal{D} \mathbf{m}_{11}^{e}}{\mathcal{D} h_{p}} & \frac{\mathcal{D} \mathbf{m}_{12}^{e}}{\mathcal{D} h_{p}} \\
\frac{\mathcal{D} \mathbf{m}_{21}^{e}}{\mathcal{D} h_{p}} & \frac{\mathcal{D} \mathbf{m}_{22}^{e}}{\mathcal{D} h_{p}}
\end{array}\right], \frac{\mathcal{D} \mathbf{m}_{j j}^{e}}{\mathcal{D} h_{p}}=\left[\begin{array}{cc}
\frac{\mathcal{D} \mathbf{m}_{11}^{e}}{\mathcal{D} h_{p}} & -\frac{\mathcal{D} \mathbf{m}_{12}^{e}}{\mathcal{D} h_{p}} \\
-\frac{\mathcal{D} \mathbf{m}_{21}^{e}}{\mathcal{D} h_{p}} & \frac{\mathcal{D} \mathbf{m}_{22}^{e}}{\mathcal{D} h_{p}}
\end{array}\right], \\
\frac{\mathcal{D} \mathbf{m}_{i j}^{e}}{\mathcal{D} h_{p}}=\left[\begin{array}{cc}
\frac{\mathcal{D} \mathbf{m}_{33}^{e}}{\mathcal{D} h_{p}} & \frac{\mathcal{D} \mathbf{m}_{34}^{e}}{\mathcal{D} h_{p}} \\
-\frac{\mathcal{D} \mathbf{m}_{43}^{e}}{\mathcal{D} h_{p}} & -\frac{\mathcal{D} \mathbf{m}_{44}^{e}}{\mathcal{D} h_{p}}
\end{array}\right], \frac{\mathcal{D} \mathbf{m}_{12}^{e}}{\mathcal{D} h_{p}}=\left(\frac{\mathcal{D} \mathbf{m}_{21}^{e}}{\mathcal{D} h_{p}}\right)^{T}, \frac{\mathcal{D} \mathbf{m}_{34}^{e}}{\mathcal{D} h_{p}}=\left(\frac{\mathcal{D} \mathbf{m}_{43}^{e}}{\mathcal{D} h_{p}}\right)^{T} .
\end{gathered}
$$

Additionally:

$$
\begin{aligned}
& \frac{\mathcal{D} \mathbf{m}_{11}^{e}}{\mathcal{D} h_{p}}=\left(\frac{\partial A_{e}}{\partial h_{p}} L_{e}+A_{e} \frac{\mathcal{D} L_{e}}{\mathcal{D} h_{p}}\right) \operatorname{diag}\left[\frac{1}{3}, \frac{13}{35}, \frac{13}{35}\right]+ \\
&+\frac{6}{5 L_{e}}\left(\operatorname{diag}\left[0, \frac{\partial J_{z e}}{\partial h_{p}}, \frac{\partial J_{y e}}{\partial h_{p}}\right]-\frac{1}{L_{e}} \frac{\mathcal{D} L_{e}}{\mathcal{D} h_{p}} \operatorname{diag}\left[0, J_{z e}, J_{y e}\right]\right), \\
& \frac{\mathcal{D} \mathbf{m}_{22}^{e}}{\mathcal{D} h_{p}}=\frac{L_{e}^{2}}{105}\left(\frac{\partial A_{e}}{\partial h_{p}} L_{e}+3 A_{e} \frac{\mathcal{D} L_{e}}{\mathcal{D} h_{p}}\right) \operatorname{diag}[0,1,1]+\frac{\mathcal{D} L_{e}}{\mathcal{D} h_{p}} \operatorname{diag}\left[\frac{1}{3} J_{x e}, \frac{2}{15} J_{y e}, \frac{2}{15} J_{z e}\right]+ \\
&+L_{e} \operatorname{diag}\left[\frac{1}{3} \frac{\partial J_{x e}}{\partial h_{p}}, \frac{2}{15} \frac{\partial J_{y e}}{\partial h_{p}}, \frac{2}{15} \frac{\partial J_{z e}}{\partial h_{p}}\right],
\end{aligned}
$$




$$
\begin{aligned}
& \frac{\mathcal{D} \mathbf{m}_{12}^{e}}{\mathcal{D} h_{p}}=\frac{11 L_{e}}{210}\left(\frac{\partial A_{e}}{\partial h_{p}} L_{e}+2 A_{e} \frac{\mathcal{D} L_{e}}{\mathcal{D} h_{p}}\right)\left[\begin{array}{ccc}
0 & 0 & 0 \\
0 & 0 & 1 \\
0 & -1 & 0
\end{array}\right]+\frac{1}{10}\left[\begin{array}{ccc}
0 & 0 & 0 \\
0 & 0 & \frac{\partial J_{z e}}{\partial h_{p}} \\
0 & -\frac{\partial J_{y e}}{\partial h_{p}} & 0
\end{array}\right], \\
& \frac{\mathcal{D} \mathbf{m}_{33}^{e}}{\mathcal{D} h_{p}}=\left(\frac{\partial A_{e}}{\partial h_{p}} L_{e}+A_{e} \frac{\mathcal{D} L_{e}}{\mathcal{D} h_{p}}\right) \operatorname{diag}\left[\frac{1}{6}, \frac{9}{70}, \frac{9}{70}\right]+ \\
& +\frac{6}{5 L_{e}}\left(\frac{1}{L_{e}} \frac{\mathcal{D} L_{e}}{\mathcal{D} h_{p}} \operatorname{diag}\left[0, J_{z e}, J_{y e}\right]-\operatorname{diag}\left[0, \frac{\partial J_{z e}}{\partial h_{p}}, \frac{\partial J_{y e}}{\partial h_{p}}\right]\right), \\
& \frac{\mathcal{D} \mathbf{m}_{44}^{e}}{\mathcal{D} h_{p}}=\frac{L_{e}^{2}}{140}\left(\frac{\partial A_{e}}{\partial h_{p}} L_{e}+3 A_{e} \frac{\mathcal{D} L_{e}}{\mathcal{D} h_{p}}\right) \operatorname{diag}[0,1,1]+\frac{1}{6}\left(\frac{\mathcal{D} L_{e}}{\mathcal{D} h_{p}} \operatorname{diag}\left[-J_{x e}, \frac{1}{5} J_{y e}, \frac{1}{5} J_{z e}\right]+\right. \\
& \left.+L_{e} \operatorname{diag}\left[-\frac{\partial J_{x e}}{\partial h_{p}}, \frac{1}{5} \frac{\partial J_{y e}}{\partial h_{p}}, \frac{1}{5} \frac{\partial J_{z e}}{\partial h_{p}}\right]\right), \\
& \frac{\mathcal{D} \mathbf{m}_{34}^{e}}{\mathcal{D} h_{p}}=\frac{13 L_{e}}{420}\left(\frac{\partial A_{e}}{\partial h_{p}} L_{e}+2 A_{e} \frac{\mathcal{D} L_{e}}{\mathcal{D} h_{p}}\right)\left[\begin{array}{ccc}
0 & 0 & 0 \\
0 & 0 & -1 \\
0 & 1 & 0
\end{array}\right]+\frac{1}{10}\left[\begin{array}{ccc}
0 & 0 & 0 \\
0 & 0 & \frac{\partial J_{z e}}{\partial h_{p}} \\
0 & -\frac{\partial J_{y e}}{\partial h_{p}} & 0
\end{array}\right] .
\end{aligned}
$$

The derivative of the inertia matrix of an element in the global system is given by the formula:

$$
\frac{\mathcal{D} \mathbf{M}_{e}}{\mathcal{D} h_{p}}=\left(\frac{\mathcal{D} \mathbf{T}_{e}}{\mathcal{D} h_{p}}\right)^{T} \mathbf{m}_{e} \mathbf{T}_{e}+\mathbf{T}_{e}^{T} \frac{\mathcal{D} \mathbf{m}_{e}}{\mathcal{D} h_{p}} \mathbf{T}_{e}+\mathbf{T}_{e}^{T} \mathbf{m}_{e} \frac{\mathcal{D} \mathbf{T}_{e}}{\mathcal{D} h_{p}}, \quad h_{p}=1, \ldots, N_{p} .
$$

On the end, the derivative of the global damping matrix $\mathbf{C}$ is equal to:

$$
\frac{\mathcal{D} \mathbf{C}}{\mathcal{D} h_{p}}=\frac{\partial \alpha}{\partial h_{p}} \mathbf{M}+\frac{\partial \beta}{\partial h_{p}} \mathbf{K}+\alpha \frac{\mathcal{D} \mathbf{M}}{\mathcal{D} h_{p}}+\beta \frac{\mathcal{D} \mathbf{K}}{\mathcal{D} h_{p}}, \quad h_{p}=1, \ldots, N_{p} .
$$

if that happens $\alpha, \beta$ depends on $\mathbf{h}$, in general. 
For derivatives of elemental matrices the aggregation process of global derivatives arrays is identical as the aggregation process for global matrices (36):

$$
\frac{\mathcal{D} \mathbf{M}}{\mathcal{D} h_{p}}=\mathrm{A}_{e} \frac{\mathcal{D} \mathbf{M}_{e}}{\mathcal{D} h_{p}}, \frac{\mathcal{D} \mathbf{K}}{\mathcal{D} h_{p}}=\mathrm{A}_{e} \frac{\mathcal{D} \mathbf{K}_{e}}{\mathcal{D} h_{p}}, \frac{\mathcal{D} \mathbf{P}}{\mathcal{D} h_{p}}=\mathrm{A}_{e} \frac{\mathcal{D} \mathbf{P}_{e}}{\mathcal{D} h_{p}}, h_{p}=1, \ldots, N_{p} .
$$

\section{Conclusions}

The DDM is simple in its mathematical form as well as in numerical implementation. The basic equation of the (12) has a similar form as the motion equation given in (2). Therefore it is easy to use the known methods of direct integrating of the equation of motion to obtain derivative of displacements, velocities and accelerations.

The algorithm of the DDM reduced to a one-time calculation of the system response via (2) and the need to integrate the equation of derivatives (12) for each parameter separately. This is a fundamental disadvantage of DDM. Because in the case of a huge model with thousands of degrees of freedom and of large number of parameters, the DDM method becomes ineffective. A helpful solution is using parallel algorithms.

In the range of civil engineering problems, where beam models predominate - so in fact with relatively small number of dynamic degrees of freedom, the DDM is attractive because of relative easy of software application.

\section{References}

[1] Adhikari S., Structural Dynamic Analysis with Generalized Damping Models. Identification, ISTE/John Wiley \& Sons, London/New York 2014.

[2] Atrek E., Virtual loads in response sensitivity analysis, In VIth AIAA/NASA/ISSMO Symposium on Multidisciplinary Analysis and Optimization, AIAA, BellevueWashington 1996.

[3] Atrek E., The virtual loads for response sensitivity analysis of nonlinear structures modeled by finite elements, In WCSMO-3, Bufallo, AIAA, New York 1999.

[4] Bathe K.-J., Finite Element Procedures, Prentice Hall, New Jersey 1998.

[5] Chen H-P., Structural Health Monitoring of Large Civil Engineering Structures, Wiley/ Blackwell, New York 2018.

[6] Choi K.K., Kim N.H., Structural Sensitivity Analysis and Optimization. Linear Systems, Mechanical Engineering Series, Springer, New York 2005.

[7] MSC Software Corporation, MSC.Marc/Mentat Manuals, v. 2018.

[8] Gopalakrishnan S., Ruzzene M., Hanagud S., Computational Techniques for Structural Health Monitoring, Springer Series in Reliability Engineering, Springer, London 2011.

[9] Hart G.C., Wong K., Structural Dynamics for Structural Engineers, John Wiley \& Sons, New York 2000. 
[10] Haug E.J., Arora J.S., Applied Optimal Design, John Wiley\&Sons, New York 1979.

[11] Holnicki-Szulc J., Virtual Distortion Method, Lecture Notes in Engineering, Springer Berlin Heidelberg 2012.

[12] Karbhari V.M., Ansari F. (ed.), Structural health monitoring of civil infrastructure systems, CRC Press, New York 2009.

[13] Kleiber M., Parameter Sensitivity in Nonlinear Mechanics, Wiley, New York 1997.

[14] Lewandowski R., Redukcja drgań konstrukcji budowlanych, PWN, 2014 (in Polish).

[15] Nocedal J., Wright S.J., Numerical Optimization, Springer, London 1999.

[16] Vanderplaats G.N., Multidiscipline Design Optimization, Vanderplaats Research \& Development, Inc., Colorado Springs 2007.

[17] Wilde K., Modal diagnostics of civil engineering structures, Gdańsk University of Technology, 2008.

[18] Xu Y-L., He J., Smart Civil Structures, CRC Press, 2017.

[19] Życzkowski M., Structural Optimization under Stability and Vibration Constrains, Springer-Verlag, Wien 1989. 